\title{
Keto microbiota: A powerful contributor to host disease recovery
}

\author{
Amanda Cabrera-Mulero ${ }^{1,2}$ - Alberto Tinahones ${ }^{1}$ - Borja Bandera ${ }^{1,2} \cdot$ Isabel Moreno-Indias $^{1,2}$. \\ Manuel Macías-González ${ }^{1,2}$. Francisco J. Tinahones ${ }^{1,2}$
}

Published online: 13 November 2019

(C) The Author(s) 2019

\begin{abstract}
Gut microbiota (GM) is a key contributor to host metabolism and physiology. Data generated on comparing diseased and healthy subjects have reported changes in the GM profile between both health states, suggesting certain bacterial composition could be involved in pathogenesis. Moreover, studies reported that reshaping of GM could contribute actively to disease recovery. Interestingly, ketogenic diets (KD) have emerged recently as new economic dietotherapeutic strategy to combat a myriad of diseases (refractory epilepsy, obesity, cancer, neurodegenerative diseases...). KD, understood in a broad sense, refers to whatever dietetic approximation, which causes physiological ketosis. Therefore, high fat-low carbs diets, fasting periods or caloric restriction constitute different strategies to produce an increase of main ketones bodies, acetoacetate and $\beta$-hydroxybutyrate, in blood. Involved biological mechanisms in ketotherapeutic effects are still to be unravelled. However, it has been pointed out that GM remodelling by $\mathrm{KD}$, from now on "keto microbiota", may play a crucial role in patient response to KD treatment. In fact, germfree animals were resistant to ketotherapeutic effects; reinforcing keto microbiota may be a powerful contributor to host disease recovery. In this review, we will comment the influence of gut microbiota on host, as well as, therapeutic potential of ketogenic diets and keto microbiota to restore health status. Current progress and limitations will be argued too. In spite of few studies have defined applicability and mechanisms of $\mathrm{KD}$, in the light of results, keto microbiota might be a new useful therapeutic agent.
\end{abstract}

Keywords Gut microbiota $\cdot$ Dysbiosis $\cdot$ Ketosis $\cdot$ Ketogenic diets $\cdot$ Keto microbiota $\cdot$ Metabolites

\section{Gut microbiota: A prokaryotic organ with multiple functions in the human body}

Gut microbiota constitute a complex and dynamic ecosystem formed by bacteria, archaea, viruses, and fungi [1]. However, bacteria are the most studied [1]. The gastrointestinal tract is one of the widest surface area in the human body [2], and probably, the most diverse microbiota within the human body [3]. Microbiota members interact between them and with the host on multiple levels.

It is known microbiota and their metabolites are able to influence our physiology, both in health and disease [3].

Manuel Macías-González

mmacias.manuel@gmail.com

1 Deparment of Endocrinology and Nutrition, Virgen de la Victoria University Hospital, Institute of Biomedical Research in Malaga (IBIMA) and University of Malaga, Campus de Teatinos $\mathrm{s} / \mathrm{n}$, 29010 Malaga, Spain

2 CIBER Physiopathology of Obesity and Nutrition (CIBERobn), Institute of Health Carlos III, Madrid, Spain
Microbiota participates in host digestion and nutrition by producing nutrients from non-digestible substrates, contributes to metabolic functions, protects against pathogens, modulates the immune system, synthetizes vitamins, and produces a wide variety of biochemically active compounds (including neurotransmitters and enzymatic cofactors) [2, 3], among others. Therefore, through these vital functions, gut microbiota influences our physiology.

Multiple metagenomic data generated on comparing feces from diseased and healthy subjects have reported changes in the gut microbiota profile between both health states. Increasing observations have described a significant correlation between an imbalance of the microbiota profile, or dysbiosis, and the development of certain diseases [1, 4]. In fact, gut microbiota changes have been defined during obesity, diabetes, liver diseases, cardiometabolic disorders, inflammatory bowel diseases, autoimmune conditions, cancer [1, 2], and even nervous system disorders (autism, anxiety, depression, multiple sclerosis, Parkinson's disease, and Alzheimer's disease) [5].

The altered microbiota profile in different diseases, as well as its putative implication in the physiopathology of these diseases, has positioned to the gut microbiota as a potential 
source of innovative therapeutics [1]. However, the symbiotic relationship between gut microbiota and its host has triggered in different profiles among individuals [3]. As result, there is not a consensus about the optimal microbiota profile, which has the potential of protecting against any disease. However, many efforts have been put for this purpose Fig. 1 .

\section{Modulation of the gut microbiota profile}

Gut microbiota composition is influenced by several factors, both genetic and environmental: human genetic, mode of delivery, type of feeding, medication (laxatives, antibiotics, proton pump inhibitors, metformin [6]), stress, infections, smoking, physical inactivity, bariatric surgery, fasting ... [7-9].

However, diet is one of the main drivers of microbiota changes [6]. The overall features of the diet (i.e., total calories, timing, variety of nutrients, vitamins and fiber ingestion, percentage of macronutrients,...) all influence the composition of the microbiota and can modify it in short time [6]. Interestingly, chrononutrition has become extremely important as modulator of microbiota, since disruption of circadian rhythms can increase the risk of disease [10]. It is known that microbiome is affected by what is eaten but also by when food is consumed [11]. Time of eating has been reported to restore circadian rhythms affecting bacterial communities and its function [10-12]. In fact, time-restricted feeding (TRF), an eating pattern in which food intake is restricted to a time window which can oscillates between 8 and $12 \mathrm{~h}[13,14]$, is able to restore the cycling of the microbiota [11]. Therefore, restoring circadian rhythms, including microbiota rhythm, may also contribute to optimize individual's physiology and decrease their risk of disease [10].

Undoubtedly, microbes that reside in the human gut are key contributors to host metabolism and, consequently, they are considered potential therapeutic targets [1]. For this reason, prebiotics, probiotics and fecal microbiota transplantation have emerged as new opportunities to promote and maintain a healthy microbiota and consequently a healthy homeostasis:

- Probiotics term refers to "administration of live microorganisms in adequate amounts able to confer a health benefit on the host". Consumption of live microbiota in both foods and dietary supplements has been related to amelioration or prevention of intestine inflammation and other intestinal or systemic disease phenotypes [15-18].

- Prebiotics are non-digestible selectively fermented dietary fibres that specifically promote the growth of beneficial targeted bacteria in gut providing some kind of benefit to the host, like immune regulation [19].

- Fecal microbiota transplantation (FMT) has been reported as a useful medical tool, for example, in treating Clostridium difficile infection or insulin-resistance in obese patients [2], but, it is necessary yet to optimize the process and make donor microbiota perdurable in receptor.

However, despite the fact diet is the strongest and safer influencer on gut bacterial communities, few clinical studies of different kind of dietary interventions on human gut microbiota have been reported so far $[2,20]$. Therefore, in this review we discuss the effect of different diet interventions on gut microbiota, focusing on a current science hot-topic, "the ketogenic diet".

\section{Keto diet and potential benefits of "keto microbiota"}

Although ketogenic diets debuted in 1920 as a medical strategy to treat refractory epilepsy, its healing properties had not been scientifically evaluated in other medical areas [21, 22]. Recently, its applicability and efficacy are being tested in the treatment of other diseases such as obesity [23], polycystic ovarian syndrome [23], cancer [24], cardiovascular problems [25] and respiratory problems [26]. Multiple worldwide open clinical trials evaluate the tolerability and efficacy of ketogenic diets in the treatment of the aforementioned pathologies as a new medical treatment (http://ClinicalTrials.gov).

$\mathrm{KD}$, understood in a broad sense, refers to any dietetic approximation able to produce a physiological ketosis, this is, an increase of ketone bodies. Classic ketogenic diets [21], fasting periods [27], time restricted feeding [13], caloric restriction diets [28], or intense physical exercise [29] constitute different strategies to produce ketonemia (increase of main ketones bodies, acetoacetate and $\beta$-hydroxybutyrate, in
Fig. 1 Gut microbiota is a key contributor to host metabolism and physiology. Ketogenic diet and/or keto microbiota might restore health state by regulating multiple mechanisms, which remain unknown. Yin Yang symbol represents host-microbial mutualism

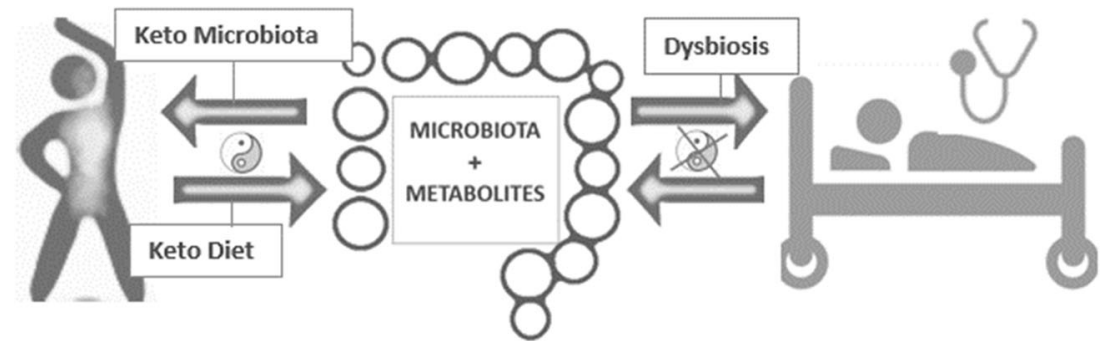


blood). Moreover, supplements that mimic the ketosis state as ketone esters [30] or ketone salts [31] have been developed in an attempt to overcome disadvantages of $\mathrm{KD}$ without a modification of the diet.

However, little is known about the underlying mechanisms of action of KD. The most accepted hypotheses point out: metabolic changes, alteration of the signalling pathways, changes in the production of hormones and neurotransmitters, epigenetic modifications [22, 24, 27], and as would be expected, modulation of the microbiota [32].

As we mentioned above, the gut microbiota plays an intermediary role between diet and host physiology. Diet affects composition, diversity and functionality of the gut microbiota and these changes in the gut microbiota are inducible and reproducible [33].

Currently, few data is available about the effects of ketogenic diets on gut microbiota composition [34]. (In this review, we have used the term "keto microbiota" to define a profile of gut microbiota moulded by a keto diet). Most of existing data about the KD impact into microbiota comes from epilepsy studies. Recent studies have reported that gut microbiota changes induced by a $\mathrm{KD}$ are required to improve the symptomatology of some diseases such as autism [35], epilepsy [33], or sclerosis [36].

\subsection{Ketogenic diet, keto microbiota and epilepsy}

The classic ketogenic diet (CKD) is a high-fat, adequate-protein, low-carbohydrate diet [21]. The most common ratio in this diet is $3: 1$ or $4: 1$. That is, $80-90 \%$ of the energy comes from fat and 10-20\% from the combination of carbohydrates and proteins [24]. The term was coined by Wilder in 1921 who found that fasting caused an improvement in their epileptic patients and tried to mimetic the ketosis state provoked by fasting with a very low carb diet [37]. Since then, CKD has been the treatment of choice in epileptic refractory patients [21].

Gut microbiota profile is significantly different between healthy and epileptic individuals. KD treatment is able to reshape gut microbiota in humans and rodents [38, 39]; and this keto microbiota is required to avoid seizures. In fact, mouse models of refractory epilepsy showed that those given antibiotics or reared in a germ-free environment were resistant to seizure protection from $\mathrm{KD}$, while keto microbiota fecal transplant helped mice with seizure control. Therefore, these results support that keto microbiota is necessary to protect against seizures $[32,40]$.

Interestingly, after a KD intervention, patients were differentiated into responder or non-responder subjects according to their gut microbiota changes, suggesting that the effectiveness of a KD was driven by the gut microbiota [5, 32]. Moreover, responder and non- responder groups differed in gut bacteria profiles at the level of order, family and genus, but also in microbial metabolites production. Such bacterial metabolites could be act by restricting precursors availability to synthetize inhibitory neurotransmitters involved in seizure control [40].

In parallel, Hampton et al. revealed that certain combinations of bacteria are required to improve epileptic symptomatology, for instance: they exposed that "Akkermansia muciniphila and Parabacteroides sp. colonization together but not alone protected against seizures in germ-free mice fed the ketogenic diet" [40]. Taken together, these findings underlie that microbiota is a complex system, where interactions between different species enable generate determined profiles of metabolites responsible to provoke a physiological response in host.

By contrast, in spite of benefits of keto microbiota in a growing number of diseases, Tagliabue reported that prolonging the KD for 3 months could cause dysmicrobism with damage to the gut health [41]. Consequently, they recommended prebiotics or probiotics treatment to re-establish gut microbiota and intestine homeostasis [41]. However, more follow-up studies are required in order to monitor the changes of the microbiota profiles with $\mathrm{KD}$, and this highlights the necessity to monitor side effects and take into account possible dysbiosis.

\section{Different ketogenic diets lead to a different gut microbiota}

Although more studies are required to compare microbiota profile between different $\mathrm{KD}$, in the light of outcome, probably, both microbiota profile and physiological responses may be dissimilar. Currently, results regarding microbial communities profile and diversity are controversial.

High fat, adequate protein, low carbs diet (CKD) seems to be associated with a lower diversity; finding which could be justified because microbiota is responsible to degrade undigested carbohydrates [2,3], which are diminished in this type of diet.

By contrast, intermittent or continuous reduction of the caloric intake, caloric restriction (CR), might not affect alphadiversity $[42,43]$. In spite of this, CR has been reported to produce significant changes in faecal bacteria composition and metabolite content $[4,43]$, thus, affecting gene expression related to metabolism and inflammation [43].

For its part, fasting periods affect clock gene transcription, [44] as well as, gut microbiota itself [45]. Disturbance of the intestinal circadian clock lead to change the uptake of nutrients, gut motility, hormones production, gut microbiota fluctuations, and ultimately, the whole body and its microbiome [43]-[45].

Main changes produced in the gut microbiota by $\mathrm{KD}$ are reflected in the Table 1 (below shown). 


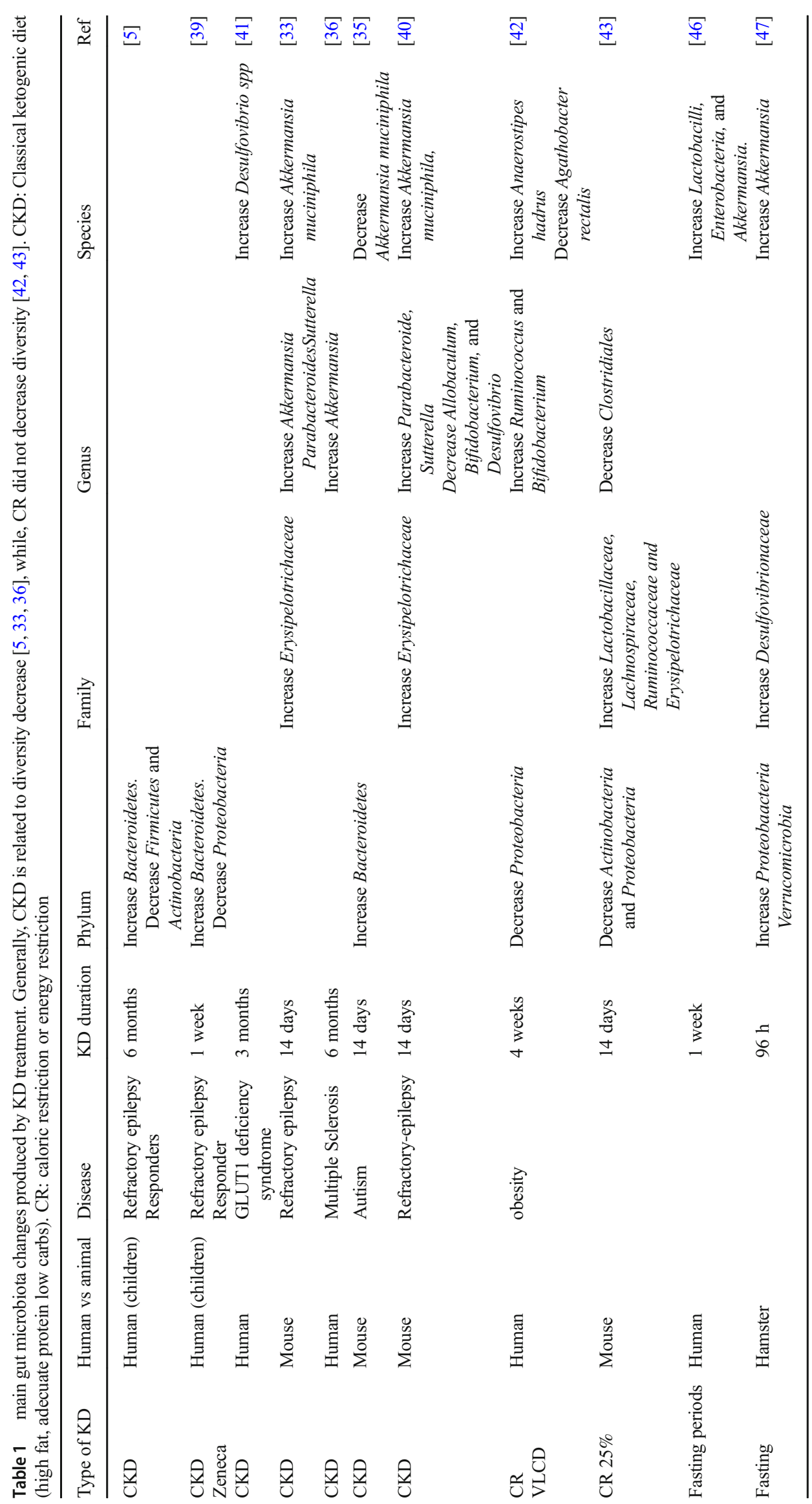


Since Zhang et al. reported different gut microbiota profile after KD between responder and non- responder epileptic patients [5], it is necessary to understand how there are different microbiota profiles more susceptible to be changed by KD than others. In addition, we must comprehend how a KD can overcome this challenge to prescribe it as a medical treatment.

\section{Benefitial effects of keto diet: Is microbiota the only responsible for?}

Previously, some studies reported that microbiota by itself was sufficient to enhance insulin sensitivity, improve tolerance to glucose and cold, and reduce fat content [48].

However, surely ketotherapeutic effects are result from different mechanisms: microbiota, epigenetic, metabolic reprogramming... Those all components could act interdependent and interrelated to many others.

Importantly, Ketone bodies (KB) fulfil several functions: 1) $\mathrm{KB}$ are energetic substrates, which are oxidized in heart, brain, and muscle during ketotic states, therefore, participating in bioenergetic homeostasis; 2) KB are also anabolic substrates contributing to lipogenesis and sterol biosynthesis in developing brain and lactating mammary gland, among other tissues;3) KB control mitochondrial metabolism and energetics; 4) KB reduce oxidative stress by inhibiting ROS/superoxide production, preventing lipid peroxidation and protein oxidation and increasing antioxidant protein levels; 5) KB act as signalling intermediators; 6) KB modulate inflammation and immune cell function; 7) KB decrease cellular damage, injury, death and lower apoptosis in neurons, lung cells and cardiomyocytes; 8) $\mathrm{KB}$ activate a reprogramming of pancreatic islet cells and could be involved in improved $\beta$-cell outcomes in states of increased ketogenesis, [49-53], and so on. Likewise, microbiota is involved in the regulation of multiple host metabolic pathways, giving rise to interactive host-microbiota metabolic, signalling, and immune-inflammatory axes that physiologically connect the gut, liver, muscle, heart, kidney and brain [54]. Therefore $\mathrm{KD}$ exert a double effect about microbiota, directly by modifying substrate availability and indirectly modifying several functions which also impacts on microbiota.

In the same line, epigenetic changes can be influenced by microbiota, but also by ketone bodies. Ketone bodies ( $\beta$ hydroxybutyrate and acetate) have been confirmed to affect epigenetic mark by inhibiting histone deacetylase class I [55], modifying proteins at the post-translational level by butyrylation [56], affecting DNA methylation [57] and acetylating histone and non-histone proteins [58]. But, metabolites produced by microbiota are also substrates or cofactors of enzymes involved in epigenetic process [59]. Therefore, it is not possible to discern epigenetic changes derived from both contributors.

Likewise, microbiota is a known endocrine organ which produces hormones and bioproducts with effects on the hosts' biology [60]. But, microbiota composition is also influenced by hormones such as estrogens [61], so, there is a mutual crosstalk. KD have shown to affect both, microbiota and several hormones levels $[13,62]$. Although to date, results about importance of gender on ketotherapeutic effects are controversial [63-65], due to the interplay between diet-microbiotahormones, the sex and hormonal status of an individual may influence on the efficacy of keto treatments. Reinforcing this, $\mathrm{KD}$ has become as a promising treatment in those diseases where sex hormones profile is altered such as ovarian or endometrial cancer [51], obesity [65] or polycystic ovarian syndrome [51].

Similarly, metabolic reprogramming may be the result of changing the microbiota profile [66], but also as a consequence of forcing to the organism to use ketone bodies like energy substrate [67]. Inflammation decrease may be result of lower content of pro-inflammatory bacteria [1], but also, consequence of lower infiltration of macrophages in adipose tissue due to fat loss derived of energy restriction [68]... so, this linking scenario could be observed with each of hypothesized mechanisms Fig. 2.

Below is a Table 2 summarising reported effects of ketotherapeutic therapies, as well as, putative involved biological mechanisms. Casual relationships are not defined yet. Additional genomics, metabolomics, and proteomics studies are required to identify molecular mechanisms through which ketosis regulates physiology responses and nuclear signalling events in each disease.

\section{Putative therapeutic scope of ketogenic diets: Keto microbiota is sought}

Ketogenic diets are being tested in degenerative and nonneurodegenerative diseases with successful results, although gut microbiota profile was not assessed. Here, we make one little sweep about last therapeutic findings of KD where it would be interesting to study keto microbiota.

\subsection{Ketogenic diet and cancer}

KD has been linked with a decrease in tumour growth [81] and an increase of the patient survival [84]. Currently, KD has been particularly suitable for adjuvant tumour therapy able to sensitize tumor cells to conventional chemotherapeutic and radiotherapeutic treatments [24, 85]. Recent studies also showed dietary administration with keto-formula could suppress tumor progression, improve systemic inmune responses and body composition, which might help to prevent cancer cachexia [82].

Probably, keto microbiota could play an important role in some kinds of cancer due to microbiome is able to establish connecting axes with other organs. 
Fig. 2 Ketogenic diets may modulate microbiota profile, epigenetic mark, and metabolic reprogramming, as well as many others. Those components could act interdependent and interrelated to many others. Exact biological mechanisms responsible for ketotherapeutic effects are still to be unravelled

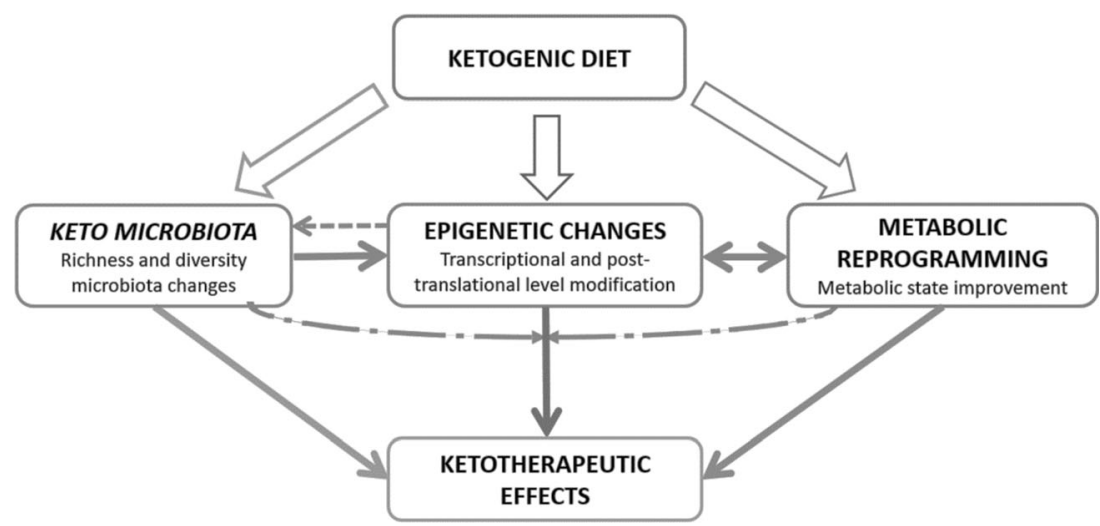

In fact, microbiota composition differs between healthy and cancer subjects, whereby, certain microbial genes can be used as cancer diagnostic biomarkers [86]. Murine experiments confirmed that germ-free mice had significant lower risk of developing colon tumours, suggesting microbiota could participate in first steps of carcinogenesis [87].

Reinforcing this, Klemment et al. underlined that there is a complex network between cancer, diet and microbiota [88]. Dietary habits have been associated with a remarkable figure of cancers [86]. The kind of consumed diet could tip the balance towards a type of microbiota able to produce oncometabolites or tumor-suppressive metabolites [89]. Both, microbiota and KD are responsible to produce short-chain fatty acids (SCFAs), whose role on cancer prevention and treatment have been broadly demonstrated. Therefore, KD and keto microbiota could contribute synergistically to prevent tumorigenesis scenario [88, 89]. Moreover, microbiota could also affect immunotherapy response and toxicity in oncological patients since microbiota modulate host immunity [90].

As a result, $\mathrm{KD}$ dietary treatment and/or keto microbiota could constitute promising strategies to curb carcinogenesis and to increase effectiveness of oncological therapies.

\subsection{Ketogenic diet and neurodegenerative diseases}

The beneficial effects of ketogenic diets have been shown in a wide variety of these neurological disorders such as Parkinson's, Alzheimer's and traumatic brain damage [71, 76]. In short, any neurodegenerative disorder characterized by neuronal hypometabolism can be approached with ketosisproducer diets since ketone bodies provide an alternative source of energy for hypometabolic neurons [91]. Similarly, it has been pointed out that $\mathrm{KD}$ could contribute to the overcoming of the cognitive deficits that occur with aging through an increase in capillary density and levels of hypoxia inducible factors [92]. Considering, gut microbiota and neuropathology are closely interrelated [36], surely defining microbiota profile will help to define a subset of neuropathologies which can be afforded by keto diet or keto microbiota.
Several studies have announced gut dysbiosis is related to neurological disease $[78,93]$. But, at the same time, gut microbiota composition can be also affected by some mental disorders [93].

At present, It is well defined the bidirectional link between neurological diseases and gut microbiota alterations [78], reinforcing the the gut-brain axis theory. Microbiota could contribute to maintain neural homeostasis by different mechanisms such as: 1) producing active biological compounds, 2) interacting with intestinal barrier, 3) connecting neuroendocrine-immune system [78], or 4) direct neuronal communication [93]. The disruption of one of these mechanisms could increase inflammation which constitutes one of the main contributors to neurodegeneration status [93].

Therefore, it is fair to say keto microbiota could play a crucial role in the ketotherapeutic responses.

(Note: Diseases mentioned in Table 2 also require analysing gut microbiota and its metabolites to shed more light about pathways and networks, which are stablished between keto microbiota and other factors).

\section{Some considerations and limitations about available literature}

- Keto microbiota resilience. So far, there is a broad lack of knowledge about remodelling capacity of microbiota after stopping KD treatment. Likewise, most studies have focused on gut microbiota. However, microbiota in other body sites could also modify by keto diet and could participate in disease recovery, so more studies are required in this field. Studying these events would contribute to improve biotherapies, even, its medical prescription to treat certain pathologies.

- Keto microbiota study follow-up. Most studies have characterized gut microbiota prior and after KD treatment. However, considering proper treatment duration is not established yet, maybe this final point of study is not representative of the most therapeutic capacity of $\mathrm{KD}$. 
Table 2 Beneficial therapeutic effects of KD in different diseases and defined biological mechanisms, which could be involved in advantages of KD

\begin{tabular}{|c|c|c|c|}
\hline Type of KD tested & Disease & Effect & $\begin{array}{l}\text { Involved biological mechanisms allegedly } \\
\text { derived by KD }\end{array}$ \\
\hline
\end{tabular}

Fasting

Classic ketogenic diet

Caloric restriction

Classic ketogenic diet

Fasting periods

Caloric restriction

Classic ketogenic diet

Classic ketogenic diet Ketone supplements

Classic ketogenic diet

Classic ketogenic diet

Fasting

Caloric Restriction

Classic ketogenic diet
Refractory epilepsy

Seizure protection $[22,39,68]$

Obesity

Weight loss and metabolic profile improvement [48, 68-73]

DT2

Improve insulin sensitivity and glycaemic control $[68,74,75]$

Alzheimer

Improve motor performance and cognition [30, 76-78]

Multiple Sclerosis

Autism

Cardiovascular

Cancer

Tumor growth decrease

Increase survival patient $[67,81-83]$

of the neurological symptoms associated with Autism Spectrum Disorder [35, 79]

Improve cardiovascular health [80]
Increase gamma-aminobutyric acid (GABA)

Decrease glutamate

$\mathrm{pH}$ alteration affecting excitability GABA

Microbiota profile reshape

Appetite suppression by ketone bodies

Gut microbiota profile and its metabolites change

Beiging of white adipose tissue

Brown adipose tissue activation (increase uncoupling protein UCP1 expression)

Loss of fat (not lean mass)

Increase metabolic cost of gluconeogenesis

Increase lipogenesis and decrease lipolysis

Metabolic improvements, browning and fat loss associated with microbiota remodelling

Decrease inflammation

Reduce insulin

Lipid profile improvement

Decrease body weight, glycaemia, glycosylated haemoglobin, triglyceride level and LDL cholesterol Increased HDL cholesterol levels

Greater weight loss than other diets in the short term

Remarkable metabolic improvement

Reduced or even withdrawn antidiabetic treatment

Compensate for the deficiency in glucose metabolism Decrease glutamate Increase GABA

Decrease reactive oxygen species (ROS)

Decrease Inflammation

Enhancing metabolism

Metabolic state improvement

Increase neurovascular integrity

Application of theory brain-gut-microbiota axis

Restore colonic microbiome

Application of theory brain-gut-microbiota axis

Application of theory brain-gut-microbiota axis

Supply of ketone bodies to the heart where KBs are efficiently oxidized

Synergic actions between butyrate production by gut bacteria and circulating high blood ketones

Application of theory gut-heart metabolic axis

Cancer cell starvation (glucose privation reduce energy production of cancer cells)

Repress Warburg effect

Decrease inflammation

Decrease insulin and insulin growing factor

Decrease angiogenesis

Decrease PI3K/Akt signalling (a known pathway

involved in carcinogenesis)

Increase apoptosis

Affect tumor epigenetic

Avoid cachexia (inhibition of muscle and body weight loss)

Sensitize cancer cells to oncological treatment 
In this sense, monitor gut microbiota periodicity could contribute to understand benefits of ketotherapy.

- Keto microbiota analysis corrected by other confusing variables: On the other hand, classic ketogenic diet (high lipids, low carbohydrates, adequate proteins) has been reported to lead to digestive problems due to the high fat content and deficiencies of minerals, vitamins and electrolytes by restringing the consumption of fruits and vegetables [94]. For this reason, vitamin and mineral supplements ingestion are recommended to alleviate symptoms and signs associated with the deficiencies of these compounds $[95,96]$. Therefore, we wonder whether studies about CKD treatment were supported with vitamins, because microbiota profile could vary based on this. Thus, microbiota changes described could not be just the result of ketosis state. Moreover, microbiota profile should be also corrected by other confusing factors (smoking, alcohol, exercise...)

- Do KD affect keto microbiota diversity? So far, few human studies have analysed changes in microbiota and its metabolites induced by $\mathrm{KD}$ and data are controversial. There are how confirm KD treatment reduce diversity, while, others affirm that there are not changes in richness and variety microbial communities. Probably, these inconsistencies are result of nonstandardized protocols and differences in KD treatment duration, age of study population, base disease, or type of KD, among others. Surely, gut microbiota changes induced by caloric restriction could be different from those that are produced by a high fat-low carbs diet. Since, different dietetic approximations influence on a diverse way, as we commented above. Therefore, the type of diet could modulate a keto microbiota, more than ketosis state per se.

- Effects of ketone supplements on microbiota: It would be interesting to test whether ketone supplements can modulate microbiota profile. This approach would represent a way to understand whether ketotherapeutic effects are just the result of ketosis or whether an additional microbial pattern is required to get these health benefits.

\section{Concluding remarks}

- Keto diet is the treatment of choice in refractory epilepsy but KD also seems to be a diet-therapeutic strategy with vigorous potential in a myriad of diseases such as cancer, metabolic and endocrine diseases and neurological disorders.

- Gut microbiota is a key contributor to host metabolism and physiology. Gut microbiota profile varies among healthy and sick individuals, and certain bacterial communities have been associated with higher risk of disease.

- KD can reshape gut microbiota profile, and this microbiota modulation is able to generate a remarkable improvement in some diseases such as epilepsy or autism.

- Ketogenic diets modulate microbiota profile, epigenetic mark, and metabolic reprogramming, as well as many other mechanisms. Those components could act interdependent and interrelated to many others.

- Further studies will confirm whether microbiota profile and ketophysiological responses varies between different ketotherapeutic strategies. Currently, results regarding keto microbial communities profile and diversity are controversial.

- Ketogenic diets together with chrononutrition may play a key role in restoring circadian rhythms and microbiota cycling, protecting from potential diseases or improving disease outcome.

- Established axis between diet-microbiota and different tissues highlights the synergic activation of different mechanisms to restore health status, reinforcing the crucial and intermediate role of microbiota for this.

In conclusion, ketogenic diets seem to be a promising new therapy, although, it is early to know its effectiveness and scope. Considering diet is the main modulator of gut microbiota, then, keto microbiota could be a key factor involved in ketotherapeutic effects since microbiome works a vital network between different organs. Nevertheless, more clinical and preclinical studies are required to define the ideal KDs exposure time, or failing that, "keto microbiota profile", to achieve proper functioning of the body in different pathologies.

Funding information This study was supported by the "Centros de Investigación Biomédica en Red" (CIBER) of the Institute of Health Carlos III (ISCIII) (CB06/03/0018), and research grants from the ISCIII (PI18/01160), and co-financed by the European Regional Development Fund (ERDF). ACM was recipient of a FPU grant from Education Minister, Madrid, Spain (Ref: FPU16/02011). IMI was supported by the Miguel Servet Type I program (CP16/00163). MMG was recipient of the Nicolas Monardes Programme from the "Servicio Andaluz de Salud, Junta de Andalucía", Spain (RC-0001-2018).

\section{Compliance with ethical standards}

Conflict of interest None of the authors have any conflict of interest to disclose.

Open Access This article is distributed under the terms of the Creative Commons Attribution 4.0 International License (http:// creativecommons.org/licenses/by/4.0/), which permits unrestricted use, distribution, and reproduction in any medium, provided you give appropriate credit to the original author(s) and the source, provide a link to the Creative Commons license, and indicate if changes were made. 


\section{References}

1. P. D. Cani, Human gut microbiome: hopes, threats and promises, Gut, vol. 67, n.o 9, pp. 1716-1725, sep. 2018.

2. E. Gianchecchi y A. Fierabracci, Recent advances on microbiota involvement in the pathogenesis of autoimmunity, Int J Mol Sci., vol. 20, no 2, p. 283, ene. 2019.

3. A. B. Shreiner, J. Y. Kao, y V. B. Young, The gut microbiome in health and in disease:, Curr Opin Gastroenterol., vol. 31, n.o 1, pp. 69-75, ene. 2015

4. X. Zheng, S. Wang, y W. Jia, Calorie restriction and its impact on gut microbial composition and global metabolism, Front Med., vol. 12, n.o 6, pp. 634-644, dic. 2018.

5. Y. Zhang, S. Zhou, Y. Zhou, L. Yu, L. Zhang, y Y. Wang, Altered gut microbiome composition in children with refractory epilepsy after ketogenic diet, Epilepsy Res., vol. 145, pp. 163-168, sep. 2018.

6. E. M. M. Quigley, Microbiota-brain-gut Axis and neurodegenerative diseases, Curr Neurol Neurosci Rep., vol. 17, n.o 12, dic. 2017.

7. M. C. Cenit, Y. Sanz, y P. Codoñer-Franch, Influence of gut microbiota on neuropsychiatric disorders, World J Gastroenterol., vol. 23, n.o 30, p. 5486, 2017.

8. M. Conlon y A. Bird, The impact of diet and lifestyle on gut microbiota and human health, Nutrients, vol. 7, n.o 1, pp. 17-44, dic. 2014.

9. C. Fraumene et al., Caloric restriction promotes rapid expansion and long-lasting increase of Lactobacillus in the rat fecal microbiota, Gut Microbes, vol. 9, n.o 2, pp. 104-114, mar. 2018.

10. E. N. C. Manoogian y S. Panda, Circadian rhythms, time-restricted feeding, and healthy aging, Ageing Res Rev., vol. 39, pp. 59-67, oct. 2017.

11. J. L. Kaczmarek, S. V. Thompson, y H. D. Holscher, Complex interactions of circadian rhythms, eating behaviors, and the gastrointestinal microbiota and their potential impact on health, Nutr Rev., vol. 75, n.o 9, pp. 673-682, sep. 2017.

12. R. M. Voigt, C. B. Forsyth, S. J. Green, P. A. Engen, y A. Keshavarzian, Circadian rhythm and the gut microbiome, en Int Rev Neurobiol, vol. 131, Elsevier, 2016, pp. 193-205.

13. M. P. Mattson, V. D. Longo, y M. Harvie, Impact of intermittent fasting on health and disease processes, Ageing Res Rev., vol. 39, pp. 46-58, oct. 2017.

14. V. D. Longo y S. Panda, Fasting, circadian rhythms, and time-restricted feeding in healthy lifespan, Cell Metab., vol. 23, n.o 6, pp. 1048-1059, jun. 2016.

15. A. Ferreiro, G. Dantas, y M. A. Ciorba, Insights into how probiotics colonize the healthy human gut, Gastroenterology, ene. 2019.

16. M. E. Sanders, A. Benson, S. Lebeer, D. J. Merenstein, y T. R. Klaenhammer, Shared mechanisms among probiotic taxa: implications for general probiotic claims, Curr Opin Biotechnol., vol. 49, pp. 207-216, feb. 2018.

17. F. Mosca, M. L. Gianni, y M. Rescigno, Can Postbiotics represent a new strategy for NEC?, New York, NY: Springer US, 2019

18. B. Sánchez, S. Delgado, A. Blanco-Míguez, A. Lourenço, M. Gueimonde, y A. Margolles, Probiotics, gut microbiota, and their influence on host health and disease, Mol Nutr Food Res., vol. 61, n.o 1, p. 1600240 , ene. 2017.

19. B. Wilson y K. Whelan, Prebiotic inulin-type fructans and galactooligosaccharides: definition, specificity, function, and application in gastrointestinal disorders: prebiotic fructans and GOS, J Gastroenterol Hepatol., vol. 32, pp. 64-68, mar. 2017.

20. B. Senghor, C. Sokhna, R. Ruimy, y J.-C. Lagier, Gut microbiota diversity according to dietary habits and geographical provenance, Hum Microbiome J., vol. 7-8, pp. 1-9, abr. 2018.

21. L. P. de B. Sampaio, Ketogenic diet for epilepsy treatment, Arq Neuropsiquiatr., vol. 74, n.o 10, pp. 842-848, oct. 2016.
22. E. H. Kossoff y J. R. McGrogan, Worldwide use of the ketogenic diet, Epilepsia, vol. 46, n.o 2, pp. 280-289, feb. 2005.

23. J. C. Mavropoulos, W. S. Yancy, J. Hepburn, y E. C. Westman, The effects of a low-carbohydrate, ketogenic diet on the polycystic ovary syndrome: a pilot study, Nutr Metab., vol. 2, p. 35, dic. 2005.

24. Allen BG, et al. Ketogenic diets as an adjuvant cancer therapy: history and potential mechanism. Redox Biol. 2014;2:963-70.

25. B. J. Brehm, R. J. Seeley, S. R. Daniels, y D. A. D'Alessio, A randomized trial comparing a very low carbohydrate diet and a calorie-restricted low fat diet on body weight and cardiovascular risk factors in healthy women, J Clin Endocrinol Metab., vol. 88, n.o 4, pp. 1617-1623, abr. 2003.

26. R. Alessandro et al., Effects of twenty days of the ketogenic diet on metabolic and respiratory parameters in healthy subjects, Lung, vol. 193, n.o 6, pp. 939-945, dic. 2015.

27. V. D. Longo y M. P. Mattson, Fasting: molecular mechanisms and clinical applications, Cell Metab., vol. 19, n.o 2, pp. 181-192, feb. 2014.

28. A. Prabhakar et al., Acetone as biomarker for ketosis buildup capability - a study in healthy individuals under combined high fat and starvation diets, Nutr J., vol. 14, n.o 1, dic. 2015.

29. P. J. Cox y K. Clarke, Acute nutritional ketosis: implications for exercise performance and metabolism, Extreme Physiol Med., vol. 3, n.o 1, p. 17, 2014.

30. M. T. Newport, T. B. VanItallie, Y. Kashiwaya, M. T. King, y R. L. Veech, A new way to produce hyperketonemia: use of ketone ester in a case of Alzheimer's disease, Alzheimers Dement., vol. 11, n.o 1, pp. 99-103, ene. 2015.

31. W. Kephart et al., The 1-week and 8-month effects of a ketogenic diet or ketone salt supplementation on multi-organ markers of oxidative stress and mitochondrial function in rats, Nutrients, vol. 9, n.o 9, p. 1019, sep. 2017.

32. E. Spinelli y R. Blackford, Gut microbiota, the ketogenic diet and epilepsy, Pediatr Neurol Briefs, vol. 32, n.o 0, p. 10, sep. 2018.

33. C. A. Olson, H. E. Vuong, J. M. Yano, Q. Y. Liang, D. J. Nusbaum, y E. Y. Hsiao, The gut microbiota mediates the anti-seizure effects of the ketogenic diet, Cell, vol. 173, n.o 7, pp. 1728-1741.e13, jun. 2018.

34. A. Paoli, G. Bosco, E. M. Camporesi, y D. Mangar, Ketosis, ketogenic diet and food intake control: a complex relationship, Front Psychol., vol. 6, feb. 2015.

35. C. Newell, M. R. Bomhof, R. A. Reimer, D. S. Hittel, J. M. Rho, y $\mathrm{J}$. Shearer, Ketogenic diet modifies the gut microbiota in a murine model of autism spectrum disorder, Mol Autism, vol. 7, n.o 1, dic. 2016.

36. A. Swidsinski et al., Reduced mass and diversity of the colonic microbiome in patients with multiple sclerosis and their improvement with ketogenic diet, Front Microbiol., vol. 8, jun. 2017.

37. J. W. Wheless, History and origin of the ketogenic diet, en Epilepsy and the ketogenic diet, C. E. Stafstrom y J. M. Rho, Eds. Totowa, NJ: Humana Press, 2004, pp. 31-50.

38. E. G. Neal et al., The ketogenic diet for the treatment of childhood epilepsy: a randomised controlled trial, Lancet Neurol., vol. 7, n.o 6, pp. 500-506, jun. 2008.

39. G. Xie et al., Ketogenic diet poses a significant effect on imbalanced gut microbiota in infants with refractory epilepsy, World J Gastroenterol., vol. 23, n.o 33, pp. 6164-6171, sep. 2017.

40. T. Hampton, Gut microbes may account for the anti-seizure effects of the ketogenic diet, JAMA, vol. 320, n.o 13, p. 1307, oct. 2018.

41. A. Tagliabue et al., Short-term impact of a classical ketogenic diet on gut microbiota in GLUT1 deficiency syndrome: a 3-month prospective observational study, Clin Nutr ESPEN, vol. 17, pp. 33-37, feb. 2017.

42. B. Ott et al., Effect of caloric restriction on gut permeability, inflammation markers, and fecal microbiota in obese women, Sci Rep., vol. 7, n.o 1, dic. 2017. 
43. K. Duszka et al., Complementary intestinal mucosa and microbiota responses to caloric restriction, Sci Rep., vol. 8, n.o 1, dic. 2018.

44. A. Mukherji, A. Kobiita, T. Ye, y P. Chambon, Homeostasis in intestinal epithelium is orchestrated by the circadian clock and microbiota cues transduced by TLRs, Cell, vol. 153, n.o 4, pp. 812 827 , may 2013.

45. R. E. Patterson y D. D. Sears, Metabolic effects of intermittent fasting, Annu Rev Nutr., vol. 37, n.o 1, pp. 371-393, ago. 2017.

46. M. Remely, B. Hippe, I. Geretschlaeger, S. Stegmayer, I. Hoefinger, y A. Haslberger, Increased gut microbiota diversity and abundance of Faecalibacterium prausnitzii and Akkermansia after fasting: a pilot study, Wien Klin Wochenschr., vol. 127, n.o 9-10, pp. 394398, may 2015.

47. K. Sonoyama et al., Response of gut microbiota to fasting and hibernation in Syrian hamsters, Appl Environ Microbiol., vol. 75, n.o 20, pp. 6451-6456, oct. 2009.

48. S. Fabbiano et al., Functional gut microbiota remodeling contributes to the caloric restriction-induced metabolic improvements, Cell Metab., vol. 28, n.o 6, pp. 907-921.e7, dic. 2018.

49. D. G. Cotter, R. C. Schugar, y P. A. Crawford, Ketone body metabolism and cardiovascular disease, AJP Heart Circ Physiol., vol. 304, n.o 8, pp. H1060-H1076, abr. 2013.

50. P. Puchalska y P. A. Crawford, Multi-dimensional roles of ketone bodies in fuel metabolism, signaling, and therapeutics, Cell Metab., vol. 25, n.o 2, pp. 262-284, feb. 2017.

51. L. Gupta, D. Khandelwal, S. Kalra, P. Gupta, D. Dutta, y S. Aggarwal, Ketogenic diet in endocrine disorders: Current perspectives, J Postgrad Med., vol. 63, n.o 4, p. 242, 2017.

52. Bolla, Caretto, Laurenzi, Scavini, y Piemonti, Low-carb and ketogenic diets in type 1 and type 2 diabetes, Nutrients, vol. 11, n.o 5, p. 962, abr. 2019

53. M. Sampson et al., $\beta$-Hydroxybutyrate improves $\beta$-cell mitochondrial function and survival, J Insul Resist., vol. 1, n.o 1, ago. 2017.

54. J. K. Nicholson et al., Host-gut microbiota metabolic interactions, Science, vol. 336, n.o 6086, pp. 1262-1267, jun. 2012.

55. T. Shimazu et al., Suppression of oxidative stress by Hydroxybutyrate, an endogenous histone deacetylase inhibitor, Science, vol. 339, n.o 6116, pp. 211-214, ene. 2013.

56. J. S. Benjamin et al., A ketogenic diet rescues hippocampal memory defects in a mouse model of kabuki syndrome, Proc Natl Acad Sci U S A., vol. 114, n.o 1, pp. 125-130, ene. 2017.

57. M. Shirahata, W.-Y. Tang, y E. W. Kostuk, A short-term fasting in neonates induces breathing instability and epigenetic modification in the carotid body, en Arterial chemoreceptors in physiology and pathophysiology, vol. 860, C. Peers, P. Kumar, C. Wyatt, E. Gauda, C. A. Nurse, y N. Prabhakar, Eds. Cham: Springer International Publishing, 2015, pp. 187-193.

58. D. M. Jaworski, A. M. A. Namboodiri, y J. R. Moffett, Acetate as a metabolic and epigenetic modifier of Cancer therapy: Acetate as a modifier of cancer therapy, J Cell Biochem., vol. 117, n.o 3, pp. 574-588, mar. 2016.

59. M. I. Bhat y R. Kapila, Dietary metabolites derived from gut microbiota: critical modulators of epigenetic changes in mammals, Nutr Rev., vol. 75, n.o 5, pp. 374-389, may 2017.

60. B. Afsar, N. D. Vaziri, G. Aslan, K. Tarim, y M. Kanbay, Gut hormones and gut microbiota: implications for kidney function and hypertension, J Am Soc Hypertens., vol. 10, n.o 12, pp. 954 961, dic. 2016.

61. J. M. Baker, L. Al-Nakkash, y M. M. Herbst-Kralovetz, Estrogengut microbiome axis: physiological and clinical implications, Maturitas, vol. 103, pp. 45-53, sep. 2017.

62. P. Sumithran et al., Ketosis and appetite-mediating nutrients and hormones after weight loss, Eur J Clin Nutr., vol. 67, n.o 7, pp. 759-764, jul. 2013.
63. A. Lyngstad et al., Investigating the effect of sex and ketosis on weight-loss-induced changes in appetite, Am J Clin Nutr., vol. 109, n.o 6, pp. 1511-1518, jun. 2019.

64. D. N. Ruskin, J. A. Fortin, S. N. Bisnauth, y S. A. Masino, Ketogenic diets improve behaviors associated with autism spectrum disorder in a sex-specific manner in the EL mouse, Physiol Behav., vol. 168, pp. 138-145, ene. 2017.

65. A. Castro et al., Effect of a very low-calorie ketogenic diet on food and alcohol cravings, physical and sexual activity, sleep disturbances, and quality of life in obese patients, Nutrients, vol. 10, n.o 10, p. 1348 , sep. 2018.

66. K. A. Krautkramer, R. S. Dhillon, J. M. Denu, y H. V. Carey, Metabolic programming of the epigenome: host and gut microbial metabolite interactions with host chromatin, Transl Res., vol. 189, pp. 30-50, nov. 2017.

67. S. K. Shukla et al., Metabolic reprogramming induced by ketone bodies diminishes pancreatic cancer cachexia, Cancer Metab., vol. 2, n.o 1, p. 18, 2014.

68. H. M. Dashti et al., Long-term effects of a ketogenic diet in obese patients, Exp Clin Cardiol., vol. 9, n.o 3, pp. 200-205, 2004.

69. A. Persynaki, S. Karras, y C. Pichard, Unraveling the metabolic health benefits of fasting related to religious beliefs: a narrative review, Nutrition, vol. 35, pp. 14-20, mar. 2017.

70. J. T. Haas y B. Staels, Fasting the microbiota to improve metabolism?, Cell Metab., vol. 26, n.o 4, pp. 584-585, oct. 2017.

71. S. Srivastava et al., Mitochondrial biogenesis and increased uncoupling protein 1 in brown adipose tissue of mice fed a ketone ester diet, FASEB J., vol. 26, n.o 6, pp. 2351-2362, jun. 2012.

72. A. Paoli, Ketogenic diet for obesity: friend or foe?, Int J Environ Res Public Health, vol. 11, n.o 2, pp. 2092-2107, feb. 2014.

73. A. Paoli, A. Rubini, J. S. Volek, y K. A. Grimaldi, Beyond weight loss: a review of the therapeutic uses of very-low-carbohydrate (ketogenic) diets, Eur J Clin Nutr., vol. 67, n.o 8, pp. 789-796, ago. 2013.

74. J. S. Volek et al., Carbohydrate restriction has a more favorable impact on the metabolic syndrome than a low fat diet, Lipids, vol. 44, n.o 4, pp. 297-309, abr. 2009.

75. A. M. Johnstone, G. W. Horgan, S. D. Murison, D. M. Bremner, y G. E. Lobley, Effects of a high-protein ketogenic diet on hunger, appetite, and weight loss in obese men feeding ad libitum, Am J Clin Nutr., vol. 87, n.o 1, pp. 44-55, ene. 2008.

76. H. M. Francis y R. J. Stevenson, Potential for diet to prevent and remediate cognitive deficits in neurological disorders, Nutr Rev., ene. 2018.

77. S. J. Koppel y R. H. Swerdlow, Neuroketotherapeutics: a modern review of a century-old therapy, Neurochem Int., jun. 2017.

78. C. Pellegrini, L. Antonioli, R. Colucci, C. Blandizzi, y M. Fornai, Interplay among gut microbiota, intestinal mucosal barrier and enteric neuro-immune system: a common path to neurodegenerative diseases?, Acta Neuropathol. (Berl.), vol. 136, n.o 3, pp. 345-361, sep. 2018.

79. Q. Li, Y. Han, A. B. C. Dy, y R. J. Hagerman, The gut microbiota and autism Spectrum disorders, front. Cell Neurosci., vol. 11, abr. 2017.

80. P. A. Crawford et al., Regulation of myocardial ketone body metabolism by the gut microbiota during nutrient deprivation, Proc Natl Acad Sci., vol. 106, n.o 27, pp. 11276-11281, jul. 2009.

81. H.-Y. Chung y Y. K. Park, Rationale, feasibility and acceptability of ketogenic diet for Cancer treatment, J Cancer Prev., vol. 22, n.o 3, pp. 127-134, sep. 2017.

82. Kentaro Nakamura, Hidekazu Tonouchi, Akina Sasayama, y Kinya Ashida, A ketogenic formula prevents tumor progression and Cancer Cachexia by attenuating systemic inflammation in Colon 26 tumor-bearing mice, Nutrients, vol. 10, n.o 2, p. 206, feb. 2018. 
83. C. L. P. Oliveira, S. Mattingly, R. Schirrmacher, M. B. Sawyer, E. J. Fine, y C. M. Prado, A nutritional perspective of ketogenic diet in Cancer: a narrative review, J Acad Nutr Diet., mar. 2017.

84. S. Khodadadi et al., Tumor cells growth and survival time with the ketogenic diet in animal models: a systematic review, Int J Prev Med., vol. 8, n.o 1, p. 35, 2017.

85. T. N. Seyfried, M. A. Kiebish, J. Marsh, L. M. Shelton, L. C. Huysentruyt, y P. Mukherjee, Metabolic management of brain cancer, Biochim Biophys Acta BBA - Bioenerg., vol. 1807, n.o 6, pp. 577-594, jun. 2011

86. K. Vipperla y S. J. O'Keefe, Diet, microbiota, and dysbiosis: a 'recipe' for colorectal cancer, Food Funct., vol. 7, n.o 4, pp. 1731-1740, 2016.

87. J. Yang y J. Yu, The association of diet, gut microbiota and colorectal cancer: what we eat may imply what we get, Protein Cell, vol. 9, n.o 5, pp. 474-487, may 2018.

88. R. J. Klement y V. Pazienza, Impact of different types of diet on gut microbiota profiles and Cancer prevention and treatment, Medicina (Mex), vol. 55, n.o 4, p. 84, mar. 2019.

89. S. J. Bultman, Interplay between diet, gut microbiota, epigenetic events, and colorectal cancer, Mol Nutr Food Res., vol. 61, n.o 1, p. 1500902, ene. 2017.

90. V. Gopalakrishnan, B. A. Helmink, C. N. Spencer, A. Reuben, y J. A. Wargo, The influence of the gut microbiome on Cancer, immunity, and Cancer immunotherapy, Cancer Cell, vol. 33, n.o 4, pp. 570-580, abr. 2018.
91. S. T. Henderson, J. L. Vogel, L. J. Barr, F. Garvin, J. J. Jones, y L. C. Costantini, Study of the ketogenic agent AC-1202 in mild to moderate Alzheimer's disease: a randomized, double-blind, placebocontrolled, multicenter trial, Nutr. Metab., vol. 6, n.o 1, p. 31, 2009.

92. L. C. Costantini, L. J. Barr, J. L. Vogel, y S. T. Henderson, Hypometabolism as a therapeutic target in Alzheimer's disease, BMC Neurosci., vol. 9, n.o Suppl 2, p. S16, 2008.

93. S. Westfall, N. Lomis, I. Kahouli, S. Y. Dia, S. P. Singh, y S. Prakash, Microbiome, probiotics and neurodegenerative diseases: deciphering the gut brain axis, Cell Mol Life Sci., vol. 74, n.o 20, pp. 3769-3787, oct. 2017.

94. P. R. Huttenlocher, A. J. Wilbourn, y J. M. Signore, Medium-chain triglycerides as a therapy for intractable childhood epilepsy, Neurology, vol. 21, n.o 11, pp. 1097-1103, nov. 1971.

95. J. W. Wheless, The ketogenic diet: an effective medical therapy with side effects, J Child Neurol., vol. 16, n.o 9, pp. 633-635, sep. 2001.

96. A. G. C. Bergqvist, Long-term monitoring of the ketogenic diet: Do's and Don'ts, Epilepsy Res., vol. 100, n.o 3, pp. 261-266, jul. 2012.

Publisher's note Springer Nature remains neutral with regard to jurisdictional claims in published maps and institutional affiliations. 